\title{
Efeitos do exercício passivo na representação cortical sensório motora: uma revisão sistemática
}

\author{
Effects of passive exercise in sensory motor cortical \\ representation: a systematic review
}

\author{
Ralf Braga Barroso ${ }^{1}$, Marília Rezende Callegari²
}

1.Fisioterapeuta. Residente pelo Programa de Residência Multiprofissional em Neurologia e Neurocirurgia/UNIFESP. São Paulo-SP, Brasil.

2.Fisioterapeuta. Mestre em Ciências/UNIFESP. Preceptora, Tutora e Coordenadora do Programa de Residência Multiprofissional em Neurologia e Neurocirurgia/UNIFESP. São Paulo-SP, Brasil.

\begin{abstract}
Resumo
Objetivo. Realizar revisão sistemática da literatura visando verificar os efeitos do exercício passivo na representação cortical sensório motora de indivíduos saudáveis ou com disfunções neurológicas, de qualquer idade. Método. Revisão sistemática de ensaios clínicos randomizados ou quasi-randomizados nas principais bases de dados (Embase, Lilacs, Medline, PEDro, Pubmed, Registro Cochrane e Scielo) que avaliaram como desfechos alterações na representação cortical sensório motora e recuperação de indivíduos após exercício passivo, sem restrição de idioma. Resultados. Foram encontrados 403 trabalhos, sendo 20 duplicados entre as bases. Destes, 30 foram classificados como potencialmente elegíveis. Após a leitura dos textos completos, somente 2 trabalhos foram incluídos. Os estudos analisados, apesar da baixa qualidade metodológica e amostras limitadas, mostraram um efeito positivo do exercício passivo nos desfechos avaliados. Conclusões. Não existem evidências suficientes para afirmar qualquer efeito do exercício passivo na representação cortical sensório motora e recuperação de habilidades sensoriais, apesar dos trabalhos incluídos terem mostrado um efeito positivo. Mais ensaios clínicos com baixo risco de viés, amostras maiores e mais homogêneas são necessários para que recomendações mais consistentes sejam realizadas.
\end{abstract}

Unitermos. Fisioterapia; Terapia por exercício; Plasticidade neuronal; Reabilitação

\begin{abstract}
Objective. To make a systematic review of the literature in order to verify the effects of passive exercise in sensory motor cortical representation of healthy individuals or individuals with neurological dysfunctions, of any age. Method. Systematic review of randomized or quasi-randomized controlled trials in the main databases (EMBASE, Lilacs, Medline, PEDro, Pubmed, Cochrane Register and Scielo) that assessed as outcomes changes in cortical sensory motor representation and participants' recovery after passive exercise, without language restriction. Results. Four hundred and three articles were found, within which 20 were duplicated between the bases. Among these, 30 were classified as potentially eligible. After reading the full text, only two articles were included. Despite the low methodological quality and limited samples, the analyzed studies showed a positive effect of passive exercise in both assessed outcomes. Conclusions. There is not enough evidence to affirm any effect of passive exercise on the cortical sensory motor representation and recovery of sensory abilities, although the included studies have shown a positive effect. More clinical trials with low risk of bias and larger and more homogeneous samples are needed so that more consistent recommendations can be made.
\end{abstract}

Keywords. Physical therapy specialty; Exercise therapy, Neuronal plasticity; Rehabilitation 


\section{INTRODUÇÃO}

O exercício passivo é definido como o movimento de um segmento corporal dentro de uma amplitude realizada inteiramente por uma força externa, podendo ser esta da gravidade, de uma máquina, de outra pessoa ou de outra parte do corpo do próprio indivíduo. Trata-se de uma intervenção fisioterapêutica extremamente utilizada em pacientes que não são capazes de movimentar um ou mais segmentos corporais, pacientes em coma, com paralisia ou totalmente restritos ao leito ${ }^{1}$.

A maior indicação clínica do exercício passivo seria evitar fibrose nas articulações após trauma ou cirurgia, prevenindo também rigidez e contraturas ${ }^{2}$. Seu benefício no pós-operatório e a curto-prazo, principalmente de cirurgias ortopédicas, tem sido estudado e evidenciado por algumas revisões sistemáticas ${ }^{3-6}$. Além disso, vale citar que algumas pesquisas vêm mostrando que 0 exercício passivo associado a protocolos de fisioterapia, incluindo também exercícios ativos, tem mostrado resultados bem mais promissores ${ }^{7}$.

A aplicação de exercícios passivos é uma intervenção bastante utilizada em indivíduos pós-acidente vascular cerebral, particularmente em estágios mais precoce do dano e em indivíduos severamente afetados ${ }^{8}$. 
Os efeitos do exercício ativo na reorganização cortical e recuperação das funções sensório-motoras são bem explicitados na literatura ${ }^{9,10}$, no entanto, o substrato neural subjacente ao incremento nestas funções provocadas por estimulação proprioceptiva repetitiva, permanece incerto ${ }^{8}$. $\mathrm{O}$ aumento na excitabilidade córtico-motora causada pelo movimento passivo levaria a uma reorganização da representação cortical do músculo alvo ${ }^{8}$.

Porém, não foi encontrada nenhuma revisão sistemática que objetivasse verificar os efeitos da movimentação passiva na reorganização cerebral. Sendo assim, o objetivo deste estudo foi realizar uma revisão sistemática da literatura sobre os efeitos do exercício passivo na reorganização cortical e representação sensório motora.

A relevância deste trabalho está no fato de que não foi encontrado nenhum estudo secundário que fornecesse evidências claras sobre a eficácia do exercício passivo na estimulação do sistema nervoso central. Os resultados deste trabalho poderão trazer questões mais objetivas que nortearão clínicos na escolha de ferramentas terapêuticas frente a um objetivo de tratamento proposto.

\section{MÉTODO}

Trata-se de uma revisão sistemática da literatura de ensaios clínicos randomizados ou quasi-randomizados. A busca eletrônica foi feita nas bases de dados Embase, 
Lilacs, Medline, PEDro, Pubmed e Scielo, de artigos publicados até outubro de 2015, com os seguintes descritores "passive exercise" OR "passive motion" conjugados com as seguintes palavras-chave "cerebral activation" OR "cortical activation" OR "neuroplasticy" sem restrição de idioma. Na base de dados PEDro foram utilizados os descritores "passive exercise" e "passive motion" e selecionados aqueles artigos potencialmente elegíveis. Na Lilacs e Medline foi usado o descritor permitido que mais se aproximasse daqueles escolhidos previamente ("terapia por exercício"). Além disso, foram utilizados como ferramenta de busca manual listas de referências de outros trabalhos encontrados e o Registro Cochrane de Ensaios Clínicos Controlados. Os artigos encontrados foram pré-selecionados com base no título e resumo, e aqueles irrelevantes para o presente trabalho foram descartados. Dois revisores analisaram os resumos e selecionaram os trabalhos potencialmente elegíveis para 0 estudo. Foram incluídos ensaios clínicos randomizados ou quasi-randomizados que objetivaram avaliar os efeitos do exercício passivo isoladamente, em algum grupo, ou em comparação com outras técnicas e/ou placebo em indivíduos saudáveis ou com disfunção neurológica, de qualquer idade. $O$ desfecho primário avaliado foram as alterações na representação sensório motora ou ativação cortical nos indivíduos estudados e o secundário foram seus efeitos na recuperação e/ou prognóstico sensório motor. 
Foram excluídos trabalhos que associaram outras técnicas com o exercício passivo em um mesmo grupo.

A extração dos dados foi realizada por meio do Software Review Manager Versão 5.3, sendo utilizado questionário de extração de dados (Anexo 1). Em caso de conflitos ou discordâncias entre os dois revisores, um terceiro foi consultado.

A qualidade dos ensaios clínicos foi avaliada pelos critérios da Ferramenta da Colaboração Cochrane para Avaliação de Risco de Viés de Ensaios Clínicos Randomizados ${ }^{11}$, sendo composta por duas partes (descrição do que foi relatado no estudo e julgamento do risco de viés para cada domínio - baixo risco de viés, alto risco de viés ou risco incerto de viés), com sete domínios: geração da sequência aleatória, ocultação da alocação, cegamento de participantes e profissionais, cegamento de avaliadores do desfecho, desfechos incompletos, relato de desfecho seletivo e outras fontes de vieses. Esta ferramenta, considerada "padrão ouro" para a avaliação de risco de viés de ensaios clínicos randomizados, não fornece uma pontuação final, mas permite um panorama geral da qualidade dos estudos incluídos na revisão sistemática prosposta $^{11,12}$.

Posteriormente a coleta de dados, os resultados dos trabalhos elegíveis foram analisados de forma qualitativa e se fosse verificado uma grande homogeneidade entre os estudos a metanálise como método estatístico para integrar estes resultados poderia ser utilizada. 
Este estudo foi aprovado pelo Comitê de Ética em Pesquisa da UNIFESP sob 0 protocolo 46305315.3.0000.5505 em julho de 2015.

\section{RESULTADOS}

A busca com os descritores nas bases de dados definidas resultou em 403 artigos, sendo que 20 estavam duplicados, resultando em um total de 383 trabalhos. Destes, somente 2 preencheram os critérios de inclusão e foram analisados nesta revisão sistemática ${ }^{13,14}$. 0 fluxograma com detalhes da busca e seleção dos estudos elegíveis pode ser visto na Figura 1.

Carel $2000^{13}$ objetivou avaliar, por meio de imagens de ressonância magnética funcional, realizadas antes e após a intervenção, se a movimentação passiva repetitiva seria capaz de modificar a representação cortical de movimentos passivos. O trabalho avaliou 14 sujeitos divididos em grupo experimental e grupo controle, mostrando que mudanças plásticas nos córtices somatossensorial e motor primários e na área motora suplementar podem ser provocadas por estimulação proprioceptiva repetitiva. 
Figura 1. Fluxograma da busca e seleção dos artigos.

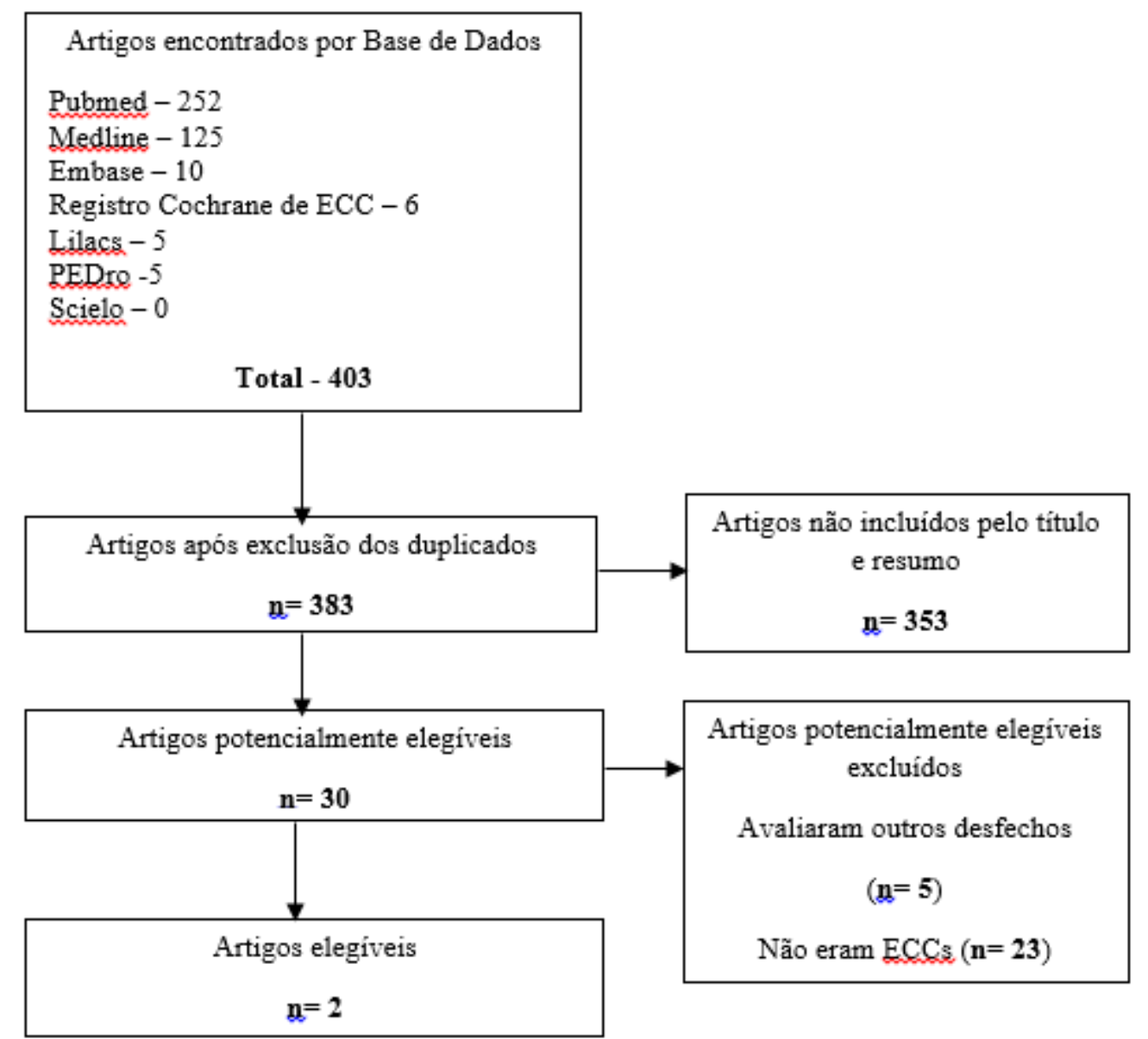

ECC: Ensaio Clínico Controlado.

Kwon $2013^{14}$ avaliou a eficácia do exercício passivo e do ativo na melhora da propriocepção, um desfecho secundário, por meio do índice de erros do ângulo de reposicionamento passivo (PAR) e ativo (AAR). No PAR, um equipamento realizava a extensão do joelho do indivíduo de $0^{\circ}$ a $90^{\circ}$ e parava aleatoriamente em uma amplitude, esperava 3 segundos para o indivíduo memorizá-la, retornava à posição inicial, realizava novamente a extensão de joelho; quando o indivíduo percebia a amplitude em que o equipamento parou da última vez, ele pressionava um botão vermelho. No AAR o indivíduo fazia ativamente a 
extensão do joelho e o examinador apertava um botão de parada aleatoriamente em uma amplitude, esperava 3 segundos, o indivíduo retornava à posição inicial e tinha que reposicionar 0 joelho na angulação em que 0 examinador apertou o botão anteriormente. Para isto, 45 sujeitos foram divididos em 3 grupos, sendo um grupo que recebeu como intervenção a movimentação passiva, outro ativa e um controle em que nenhum exercício foi realizado. O grupo que recebeu movimentação passiva obteve melhores resultados no índice de erros tanto do PAR quanto do AAR. A Tabela 1 traz as características e os resultados dos artigos selecionados.

A análise da qualidade dos trabalhos estudados mostrou que ambos apresentaram a classificação de risco incerto de viés na maioria dos itens da Ferramenta da Colaboração Cochrane para Avaliação de Risco de Viés de Ensaios Clínicos Randomizados, ${ }^{11}$ conforme aponta a Tabela 2. O dois estudos ${ }^{13,14}$ obtiveram a classificação de risco incerto de viés em 5 itens e em somente dois, baixo risco.

Além disto, estes trabalhos apresentaram amostras muito limitadas fazendo com que a força das recomendações advindas deles seja mais baixa.

Devido à heterogeneidade dos desfechos analisados e da amostra entre os estudos, a comparação entre eles não pode ser feita e, portanto, a metanálise não pode ser realizada para uma avaliação quantitativa. 
Tabela 1. Características dos estudos selecionados.

\begin{tabular}{|c|c|c|c|c|c|}
\hline Estudo & $\begin{array}{c}\text { n/mulheres } \\
(\%)\end{array}$ & Sujeitos & $\begin{array}{c}\text { Avaliação } \\
\text { do } \\
\text { Desfecho }\end{array}$ & Intervenção & Resultados \\
\hline $\begin{array}{c}\text { Carel } \\
2000^{13}\end{array}$ & $\begin{array}{l}I-6 \\
C-6 \\
58 \%\end{array}$ & Saudáveis & $\begin{array}{c}\text { Imagens de } \\
\text { ressonância } \\
\text { magnética } \\
\text { funcional }\end{array}$ & $\begin{array}{l}\text { I - } \\
\text { flexão/extensão } \\
\text { passiva de } \\
\text { punho direito, } \\
20 \text { min/dia, } 5 \\
\text { dias/sem, } 4 \\
\text { sem } \\
\text { C - nenhuma } \\
\text { intervenção }\end{array}$ & $\begin{array}{l}\text { Mudanças } \\
\text { plásticas na } \\
\text { representação } \\
\text { cortical no } \\
\text { grupo } \\
\text { intervenção }\end{array}$ \\
\hline $\begin{array}{l}\text { Kwon } \\
2013^{14}\end{array}$ & $\begin{array}{l}I-15 \\
O-15 \\
C-15 \\
44,4 \%\end{array}$ & $\begin{array}{c}\text { AVC } \\
\text { (Hemiparesia) }\end{array}$ & $\begin{array}{l}\text { Índice de } \\
\text { Erro do PAR } \\
\text { e AAR }\end{array}$ & $\begin{array}{l}\text { I - } \\
\text { Flexão/extensão } \\
\text { passiva de } \\
\text { joelho de } 10^{\circ} \text { a } \\
100^{\circ}, 60 \text { vezes } \\
\text { com velocidade } \\
120^{\circ} \\
0- \\
\text { Flexão/extensão } \\
\text { passiva de } \\
\text { joelho de } 10^{\circ} \text { a } \\
100^{\circ}, 60 \text { vezes } \\
\text { com velocidade } \\
120^{\circ} \text { com um } \\
\text { programa de } \\
\text { exercício } \\
\text { isocinético ativo } \\
\text { C - Nenhuma } \\
\text { intervenção }\end{array}$ & $\begin{array}{l}\text { Movimentação } \\
\text { passiva foi a } \\
\text { melhor } \\
\text { estratégia } \\
\text { para melhorar } \\
\text { a } \\
\text { propriocepção. }\end{array}$ \\
\hline
\end{tabular}

AAR: ângulo de reposicionamento ativo; AVC: acidente vascular cerebral; C: controle; I: intervenção; O: outra intervenção; PAR: ângulo de reposicionamento passivo.

Tabela 2. Análise da qualidade dos estudos incluídos.

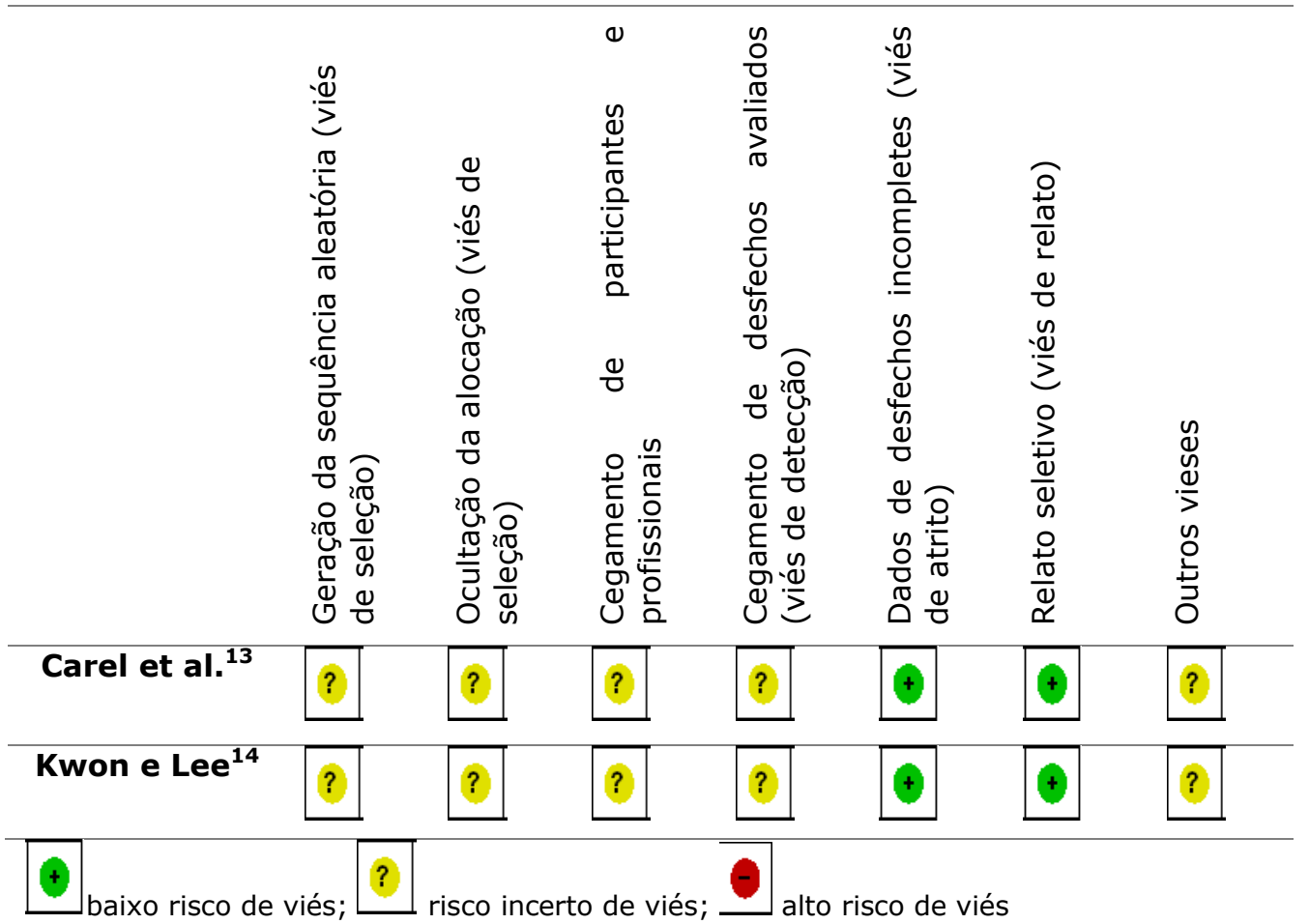




\section{DISCUSSÃO}

As revisões sistemáticas e metanálises são consideradas os melhores métodos para resumir evidências sobre a eficácia das intervenções de saúde. Os métodos sistemáticos são utilizados para evitar vieses e possibilitar uma análise mais objetiva dos resultados relacionados a uma intervenção específica. Porém, é importante lembrar que este é um tipo de estudo secundário, isto é, desenhado e conduzido após a publicação de muitos estudos experimentais sobre o tema, desta forma, uma revisão sistemática depende da qualidade da fonte primária ${ }^{15,16}$.

O resultado desta revisão sistemática, apesar do número limitado de trabalhos elegíveis, assim como suas qualidades e tamanho de suas amostras, é capaz de evidenciar uma tendência de que o exercício passivo pode gerar alterações plásticas na representação cerebral sensorial e motora e interferir positivamente na recuperação dos indivíduos que não são capazes de realizar movimentação voluntária.

Nenhum estudo secundário que avaliasse o efeito do exercício passivo nos desfechos analisados pelo presente estudo foi encontrado, desta maneira, não foi possível confrontar os resultados desta pesquisa com outras. Uma possível justificativa é escassez de estudos primários sobre o assunto. No entanto, alguns trabalhos do tipo séries de caso $^{17-19}$ visaram avaliar os efeitos do exercício passivo sobre a ativação cerebral. Os trabalhos de Sternueuagel et $a l^{17}$ e de Doering et $a l^{19}$ avaliaram o efeito do exercício 
ativo e do passivo sobre a média e o pico de velocidade do fluxo sanguíneo na artéria cerebral média, uma medida indireta do metabolismo no tecido cerebral e sua consequente ativação, concluindo que tanto o exercício passivo quanto o ativo foram capazes de aumentar a velocidade deste fluxo. O estudo de Pittaccio et $a l^{18}$ avaliou a ativação cortical por meio da eletroencefalografia e espectroscópio infravermelho mostrando que o movimento voluntário e o passivo/assistido apresentaram na comparação entre os mapas gerados um padrão de ativação similar. Isto é um achado importante, pois permite-se inferir que o exercício passivo é capaz de ativar aferências somatossensoriais que são processadas da mesma maneira que as ações voluntárias. Da mesma forma, por meio de estimulação magnética transcraniana, estudou-se o efeito sobre a área e o volume da representação cortical do músculo flexor do punho em indivíduos saudáveis e hemiplégicos após $30 \mathrm{~min}$ de flexão/extensão passiva de punho ${ }^{8}$. Foi observado um aumento no volume do mapa desta representação cortical em indivíduos saudáveis somente.

Porém, estes achados devem ser interpretados com cautela, primeiro pelo fato dos estudos citados acima, bem como os descritos nesta revisão, apresentarem amostras pequenas, além de serem compostas em sua maioria por indivíduos saudáveis, o que dificulta extrapolar tais achados para outras populações. Além disto, os resultados dos estudos do tipo séries de caso, por não possuir um grupo 
controle, podem ser atribuídos ao acaso, fornecendo desta maneira, um grau de evidência mais baixo. Seria possível realizar uma metanálise com as séries de caso e avaliar se os resultados, mesmo que sem um grupo controle, apresentam um desfecho positivo, mas para isso seria necessário refazer as buscas e incluir possíveis estudos excluídos inicialmente pelo tipo de estudo, caracterizando um viés nesta revisão sistemática. Foram observadas diferenças nos resultados entre duas ressonâncias magnéticas funcionais em espaços de tempo distintos nos mesmos sujeitos, informação de extrema relevância para este contexto, visto que muitos estudos sobre os efeitos da movimentação passiva na ativação cerebral utilizam este método ${ }^{20}$.

Os dois ensaios clínicos controlados analisados ${ }^{13,14}$ neste trabalho, além de suas limitações no tamanho da amostra e na avaliação do risco de viés, avaliaram populações e desfechos distintos e utilizaram métodos diferentes, o que impossibilita, por sua vez, agrupar estes dados em uma única análise. Cabe citar também, que o protocolo e a articulação escolhida em cada estudo foram diferentes, dificultando uma recomendação clínica de frequência, intensidade e duração da intervenção capaz de produzir algum efeito.

Outro ponto importante a se discutir é a relevância clínica da estimulação proprioceptiva repetitiva na recuperação de habilidades funcionais e deve ser objeto de estudo de outros ensaios clínicos controlados, pois qualquer 
estímulo, seja proprioceptivo, tátil, térmico ou doloroso, fisiologicamente, gera processamento a nível cortical e subcortical ${ }^{21,22}$, porém, nada se sabe sobre os efeitos desta estimulação no prognóstico motor de indivíduos que não são capazes de realizar movimento ativo. Além disto, futuros ensaios clínicos devem focar em amostras maiores, padronização de protocolos e instrumentos de medida e no baixo risco de viés, para que outras revisões sistemáticas tenham subsídios para realizar recomendações mais consistentes sobre protocolos e eficácia tanto do ponto de vista da ativação de caminhos adjacentes ao processamento sensório motor, quanto de sua relevância clínica para recuperação e prognóstico motor dos indivíduos.

\section{CONCLUSÃO}

Não existem evidências suficientes para afirmar um efeito do exercício passivo sobre a representação cortical sensório-motora, mesmo os dois trabalhos analisados mostrando um efeito positivo. Faltam ensaios clínicos controlados com amostras maiores e mais homogêneos para que uma análise estatística da eficácia desta intervenção e recomendações clínicas sobre o melhor protocolo de estimulação possam ser realizadas. Estudos futuros devem focar em entender também os efeitos da movimentação passiva na recuperação funcional e 
prognóstico motor de indivíduos com disfunções

neuromotoras.

\section{REFERÊNCIAS}

1.Kisner C, Colby LA. Range Motion. In: Kisner C, Colby LA. Therapeutic Exercise: foundations and tecniques. $5^{a}$ ed. Philadelfia: FA Dalvis Company, 2007, p.44-64.

2.O'Driscoll SW, Giori, NJ. Continuous passive motion (CPM): theory and principles of clinical application. J Rehab Res Dev 2000;37:17988.

3.Viswanathan $\mathrm{P}$, Kidd $\mathrm{M}$. Effect of continuous passive motion following total arthroplasty on knee range of motion and function: a systematic review. NZ J Physioter 2010;38:14-22.

4.Farsetti P, Caterini R, Potenza V, Luna V, Maio F, Ippolito E. Immediate passive continuous motion after internal fixation of an ankle fracture. J Orthop Traumatol 2009;10:63-9. https://doi.org/10.1007/s10195-009-0048-4

5.Grella RJ. Continuous passive motion following total knee arthroplasty: a useful adjunct to early mobilisation? Physl Ther Rev 2008;13:269-79. https://doi.org/10.1179/174328808X309197

6. Milne S, Brosseau L, Robinson V, Noel MJ, Davis J, Drouin $\mathrm{H}$, et al. Continuous passive motion following total knee arthroplasty. Cochrane Database Syst Rev 2003;CD004260. https://doi.org/10.1002/14651858.CD004260

7.Mau-Moeller A, Behrens M, Finze S, Bader R, Mittelmeier. The effect of the continuous passive motion and sling exercise training on clinical and functional outcomes following total knee arthroplasty: a randomized active-controlled clinical study. Healthy Qual Life Outcomes 2004;12:1-10. https://doi.org/10.1186/1477-7525-12-68

8.Lewis GN, Byblow WD. The effects of repetitive proprioceptive stimulation on corticomotor representation in intact and hemiplegic individuals. Clin Neurophysiol 2004;115:765-73. https://doi.org/10.1016/j.clinph.2003.11.014

9.Thrane G, Friborg O, Anke A, Indreday B. A meta-analysis of constraint-induced movement therapy after stroke. J Rehabil Med 2014;46:833-42. https://doi.org/10.2340/16501977-1859

10.Shumway-Cook A, Woollacott MH. Controle Motor: discussões e teorias. In: Shumway-Cook A, Woollacott MH. Controle Motor: teorias e aplicações práticas. $3^{a}$ ed. Barueri: Manole, 2010, p3-20.

11. Higgins JPT, Green S (ed). Cochrane Handbook for Systematic Reviews of Interventions Version 5.1.0 [update March 2011]. The Cochrane Collaboration 2011.

12. Carvalho APV, Silva V, Grande AJ. Avaliação do risco de viés de ensaios clínicos randomizados pela ferramenta da colaboração Cochrane. Diagn Tratamento 2013;18:38-44. 
13. Carel C, Loubinox I, Boulanouar K, Manelfe C, Rascol O, Celsis P, et al. Neural substracte for the effect of passive training on sensorimotor cortical representation: a sutdy with functional magnetic resonance imaging in healthy subjects. J Cereb Flow Met 2000;20:478-84. https://doi.org/10.1097/00004647-20000300000006

14.Kwon OS, Lee SW. Effect of continuing repreated passive and active exercises on knee's position senses in patients with hemiplegia. NeuroRehabilitation 2013;33:391-7. https://doi.org/10.3233/NRE-130969

15.Sampaio RF, Mancini MC. Estudos de revisão sistemática: uma guia para a síntese criteriosa da evidência científica. Rev Bras Fisioter São Carlos 2007;11:83-9.

http://dx.doi.org/10.1590/S1413-35552007000100013

16.Linde K, Willich SN. How objective are systematic reviews? Differences between reviews on complementary medicine. J R Soc Med 2003;96:17-22. http://dx.doi.org/10.1258/jrsm.96.1.17

17.Pittaccio S, Garavaglia L, Molteni E, Guanziroli E, Zappasodi F, Beretta $\mathrm{E}$, et al. Can passive mobilization provide clinically-relevant brain stimulation? A pilot EEG and NIRS study on healthy subjects. Eng Med Biol Soc 2013;2013:3547-50. http://dx.doi.org/10.1109/EMBC.2013.6610308

18.Steuernagel B, Brix J, Schneider B, Fischer GC, Doering TJ. Effects of active and passive movement stimuli on cerebral hemodynamics and the cerebral metabolism. Forsch Komplementarmed Klass Naturheilkd 2002;9:331-7. http://dx.doi.org/10.1159/000069232

19.Doering TJ, Resch KL, Steuernagel B, Brix J, Schneider B, Fischer GC. Passive and active exercises increase cerebral blood flow velocity in young, healthy individuals. Am J Phys Med Rehabil 1998;77:490-3. http://dx.doi.org/10.1097/00002060-199811000-00006

20.Loubinox I, Boulanouar K, Ranjeva JP, Carel C, Berry I, Rascol O, et al. Cerebral fMRI activation modulated by a single dose of the monoamines neurotransmission enhancers fluoxetine and fenozolone during sensorimotor tasks. J Cereb Blood Flow Metab 1999;19:136575. http://dx.doi.org/10.1097/00004647-199912000-00010

21.Bear MF, Connors BW, Paradiso MA. O sistema sensorial somático. In: Bear MF, Connors BW, Paradiso MA. Neurociências: desvendando o sistema nervoso. $2^{a}$ edição. Angélica Rosat Consiglio. Porto Alegre: Artmed, 2002, 397-436.

22.Bear MF, Connors BW, Paradiso MA. Controle espinhal do movimento. In: Bear MF, Connors BW, Paradiso MA. Neurociências: desvendando o sistema nervoso. $2^{a}$ edição. Aldo Bolten Lucion. Porto Alegre: Artmed, 2002, 437-65. 
Anexo 1. Formulário de extração de dados

Revisão Sistemática: Efeitos do exercício passivo na representação cortical sensório motora: uma revisão sistemática

\section{Reviewers name}

\section{Study ID - First Author, Date of Publication, Title, Place of Publication}

\section{CLASSIFICATION: （ ) Study included $\quad(\quad$ ) Study exclude with reasons:}

( ) Study awaiting classification with reason:

\section{Study design}

( )clinical trial ( )Parallel group ( )Crossover ( )Observational ( )Case study ( )Case series ( )Systematic review ( )Other (describe)

Is the treatment allocation described as RANDOMISED /quasi-randomised? ( )Yes ( )No; Informed ( ) yes ( ) no

\section{Methods:}

\section{Participants:}

Diagnostic criteria fulfilled?

\section{Other (describe):}

Exclusion criteria

Associação do exercício passivo com outras técnicas no mesmo grupo

( )Yes ( ) No ( ) Not clear

Other causes excluded?

( ) Yes ( ) No ( ) Not clear

Gender: ( ) males and ( ) females)

Age: median _ years (_ to _, SD _ $)$

Race:

Setting:

\section{Participants distribution:}

\begin{tabular}{|l|l|l|l|l|}
\hline & $\begin{array}{l}\text { Intervention } \\
(\mathbf{n})\end{array}$ & $\begin{array}{l}\text { Other } \\
\text { treatment }\end{array}$ & Placebo & $\begin{array}{l}\text { No } \\
\text { treatment }\end{array}$ \\
\hline $\begin{array}{l}\text { Total number participants } \\
(\mathrm{N}=\quad \text { ) }\end{array}$ & & & & \\
\hline
\end{tabular}

Completeness of follow-up

\begin{tabular}{|l|l|l|l|l|}
\hline & Intervention & $\begin{array}{l}\text { Other } \\
\text { treatment }\end{array}$ & placebo & $\begin{array}{l}\text { No } \\
\text { treatment }\end{array}$ \\
\hline Total number randomised & & & & \\
\hline Number of withdrawals & & & & \\
\hline Reason for withdrawals & & & & \\
\hline Number lost to follow up & & & & \\
\hline $\begin{array}{l}\text { Final number of patients } \\
\text { evaluated }\end{array}$ & & & & \\
\hline
\end{tabular}

Intervention details:

Type of intervention

Timing/frequency/dose

Duration of treatment

Risk of Bias table-Assessment of methodological quality of trials that meet the inclusion criteria: Support for judgement

Random sequence generation ( ) high risk ( ) low risk

Allocation concealment ( ) high risk ( ) low risk

Blinding of participants ( ) high risk ( ) low risk

Blinding of outcome assessment( ) high risk ( ) low risk

( ) unclear risk

( ) unclear risk

( ) unclear risk

( ) unclear risk 
Incomplete outcome data Selective reporting Other bias

$\begin{array}{lll}\text { ( ) high risk } & \text { ( ) low risk } \\ \text { ( ) high risk } & (\text { ) low risk } \\ \text { ( ) high risk } & \text { ( ) low risk }\end{array}$

( ) unclear risk

( ) unclear risk

( ) unclear risk

GRADE quality of evidence:

\begin{tabular}{|c|l|l|l|}
\hline Questions & No & Serious (-1) & $\begin{array}{c}\text { Very serious } \\
(\mathbf{- 2})\end{array}$ \\
\hline Limitations in design? & & & \\
\hline Inconsistency? & & & \\
\hline Indirectness? & & & \\
\hline Imprecision & & & \\
\hline Publications bias & & & \\
\hline
\end{tabular}

Results

Outcomes measures:

Primary outcome measure

( ) reorganização ou ativação da representação cortical sensório-motora

\section{Secondary outcome measures}

( ) Melhora na recuperação

Instructions: Choose one table below according the study outcome or edit it with the study assessment and filling in with the results

\begin{tabular}{|l|l|l|l|l|l|}
\hline & \multicolumn{2}{|c|}{ Intervention } & \multicolumn{2}{c|}{ Control } & $\begin{array}{l}\text { Notes/Additional } \\
\text { data/Missing } \\
\text { data }\end{array}$ \\
\hline & $\begin{array}{l}\text { Number } \\
\text { of } \\
\text { incomple } \\
\text { te } \\
\text { recovery }\end{array}$ & $\begin{array}{l}\text { Total } \\
\text { num } \\
\text { ber }\end{array}$ & $\begin{array}{l}\text { Number } \\
\text { of } \\
\text { incompl } \\
\text { ete } \\
\text { recover } \\
\text { yotal }\end{array}$ & $\begin{array}{l}\text { Tor } \\
\text { numb } \\
\text { er }\end{array}$ & \\
\hline $\begin{array}{l}\text { Houve } \\
\text { ativação/reorgan } \\
\text { ização }\end{array}$ & & & & & \\
\hline $\begin{array}{l}\text { Não houve } \\
\text { ativação/reorgan } \\
\text { ização }\end{array}$ & & & & & \\
\hline
\end{tabular}

Adverse effects attributable to the treatment (all patients)

\begin{tabular}{|l|l|l|l|l|l|}
\hline & \multicolumn{2}{|c|}{ Intervention } & \multicolumn{2}{c|}{ Control } & $\begin{array}{l}\text { Notes/Additional } \\
\text { data/Missing data }\end{array}$ \\
\hline & $\begin{array}{l}\text { Number } \\
\text { experiencing } \\
\text { event }\end{array}$ & $\begin{array}{l}\text { Total } \\
\text { number } \\
\text { in group }\end{array}$ & $\begin{array}{l}\text { Number } \\
\text { experiencing } \\
\text { event }\end{array}$ & $\begin{array}{l}\text { Total } \\
\text { number } \\
\text { in group }\end{array}$ & \\
\hline $\begin{array}{l}\text { Any adverse event discussed } \\
\text { in the trial }\end{array}$ & & & & & \\
\hline Adverse event A (specify) & & & & & \\
\hline Adverse event B (specify) & & & & & \\
\hline
\end{tabular}

Text entry for main conclusions of the trial 